doi: http://dx.doi.org/10.7124/bc.0009BC

\section{mTORC1 pathway in DNA damage response}

Yinxing Ma ${ }^{1}$, Licia Silveri ${ }^{1}$, John LaCava ${ }^{2}$, Yegor Vassetzky $^{1}$, Svetlana Dokudovskaya ${ }^{1}$

${ }^{1}$ CNRS UMR 8126, Université Paris-Sud, Gustave Roussy, 114, rue Edouard Vaillant, 94805, Villejuif, France; ${ }^{2}$ Laboratory of Cellular and Structural Biology, The Rockefeller University, New York, New York, USA.

s.dokud@gmail.com

Living organisms have evolved various mechanisms to control their metabolism and response to various stresses, allowing them to survive and grow in different environments. In eukaryotes, the highly conserved mechanistic target of rapamycin (mTOR) signaling pathway integrates both intracellular and extracellular signals and serves as a central regulator of cellular metabolism, proliferation and survival. A growing body of evidence indicates that mTOR signaling is closely related to another cellular protection mechanism, the DNA damage response (DDR). Many factors important for mTOR pathway are also involved in the DDR. The SEA/GATOR complex is an inhibitor of the mTORC1 pathway. In mammals the GATOR1 complex is composed of the proteins DEPDC5, NPRL2 and NPRL3. We characterized mammalian cells overexpressing the GATOR1 component NPRL2 and found that in the cells with active p53, ectopic expression of NPRL2 induces NOX2-dependent production of reactive oxygen species and DNA damage. Overexpressed NPRL2 accumulates in the nucleus, together with apoptosis-inducing factor (AIF). These events are accompanied by phosphorylation of $\mathrm{p} 53$, activation of a DNA-damage response and cell cycle arrest in G1 phase, followed by apoptosis. In the cells negative for active $\mathrm{p} 53$, NPRL2 ectopic expression leads to activation of $\mathrm{CHK} 1$ or $\mathrm{CHK} 2$ kinases and cell cycle arrest in $\mathrm{S}$ or G2/M phases. Combined, these results demonstrate a new role for the NPRL2, distinct from its function in mTORC1 regulation.

doi: http://dx.doi.org/10.7124/bc.0009BD

\section{ING3 is required for ATM signaling and DNA repair in response to DNA double strand breaks}

Audrey Mouche ${ }^{1}$, Jérôme Archambeau ${ }^{1}$, Charles Ricordel ${ }^{1}$, Laura Chaillot ${ }^{1,4}$, Nicolas Bigot ${ }^{2,3}$, Thierry Guillaudeux ${ }^{1,4}$, Muriel Grenon ${ }^{6}$ and Rémy Pedeux ${ }^{1 *}$

${ }^{1}$ INSERM U1242, COSS, Université de Rennes 1, CLCC Eugène Marquis, Rennes, France; ${ }^{2}$ INSERM U1236, MICMAC, Rennes, France; ${ }^{3}$ Present address: Genome Damage and Stability Centre, University of Sussex, Falmer, Brighton BN1 9RQ, UK; ${ }^{4}$ UMS Biosit, SFR Biologie-Santé, Rennes; ${ }^{5}$ Biochemistry, School of Natural Sciences, National University of Ireland, Galway, Ireland remy.pedeux@univ-rennes1.fr

ING3 (Inhibitor of Growth 3) is a candidate tumor suppressor gene whose expression is lost in tumors. Aims: We want to identify and characterize new tumor suppresor functions for ING3. Methods: We conduct experiments in yeast and human cells depleted or not for ING3 and exposed to genotoxic agents. Results: ING3-depleted human cells and yeast cells deleted for its ortholog YNG2 are sensitive to DNA damage suggesting a conserved role in response to such stress. - In human cells, ING3 
is recruited to DNA double strand breaks and is required for ATM activation. - In response to doxorubicin, ATM activation is dependent on ING3 but not on TIP60, whose recruitment to DNA breaks also depends on ING3. - These events lead to ATM-mediated phosphorylation of NBS1 and of major mediators of the DNA damage response. - Upon genotoxic stress, DNA repair by Non Homologous End Joining (NHEJ) or Homologous Recombination (HR) were impaired in absence of ING3. - Immunoglobulin Class Switch Recombination (CSR), a physiological mechanism requiring NHEJ repair, was impaired in the absence of ING3. Conclusions: Since deregulation of DNA double strand break repair is associated with genomic instability, we propose a novel function of ING3 as a caretaker tumor suppressor involved in the DNA damage signaling and repair.

A.M. was a recipient of a doctoral fellowship from $\mathrm{La}$ Ligue Contre le Cancer and Region Bretagne. R.P. \& M.G. were recipients of from Ulysses "The France - Ireland Exchange Scheme". N. Bigot was a recipient of ANR program (SAFE 2012) (ANR-11-RPIB-0012). C.

References: Cell Death Differ. 2019 Feb 25. doi: 10.1038/s41418-019-0305-x. [Epub ahead of print]

doi: http://dx.doi.org/10.7124/bc.0009BE

\section{Computer modeling of phase separation at PML nuclear bodies}

Richard Henze, Marvin Wenzel, Peter Dittrich \& $\underline{\text { Peter Hemmerich }}$

Leibniz Institute on Aging

peter.hemmerich@leibniz-fli.de

The biophysical mechanism of liquid-liquid phase separation (LLPS) has emerged as an attractive idea to explain the formation and function of membrane-less organelles, such as nuclear bodies. Our aim is to simulate the genesis of promyelocytic leukemia nuclear bodies (PML-NBs) at the molecular level using computer models in order to gain insight on LLPS within PML-NBs. To this end, we have generated a computer model of PML-NB assembly which uncovers molecular details of their genesis and a spatial map of its LLPSdriving elements. Model predictions can be exploited to test new hypotheses of PML-NB structure in the wet lab in an iterative process. The established computer model may be expanded to other membrane-less organelles to reveal their structural details.

doi: http://dx.doi.org/10.7124/bc.0009BF

\section{Interplay of DNA replication, repair and chromatin}

M. Cristina Cardoso

Cell Biology and Epigenetics, Dept. Biology, TU

Darmstadt, Germany

cardoso@bio.tu-darmstadt.de

Half a century ago the elucidation of the DNA double helix structure was quickly followed by the visualization of replicons in DNA fibers. To connect 1D DNA replication/repair information with whole cell 3D data in mammalian cells, we combined super resolution microscopy and time-lapse analysis of S-phase dynamics with genome size and DNA replication fiber analysis. We found that the subnuclear replication structures can be optically resolved down to single replicons during all S-phase stages. This sets aside the conventional inter- 